\title{
Effects of thermohaline instability and rotation-induced mixing on the evolution of light elements in the Galaxy: D, ${ }^{3} \mathrm{He}$ and ${ }^{4} \mathrm{He}$
}

\author{
N. Lagarde ${ }^{1}$, D. Romano ${ }^{2}$, C. Charbonnel ${ }^{1,3}$, M. Tosi ${ }^{2}$, C. Chiappini ${ }^{4}$, and F. Matteucci ${ }^{5,6}$ \\ 1 Geneva Observatory, University of Geneva, Chemin des Maillettes 51, 1290 Versoix, Switzerland \\ e-mail: Nadege. Lagarde@unige.ch \\ 2 INAF - Bologna Observatory, via Ranzani 1, 40127 Bologna, Italy \\ 3 IRAP, CNRS UMR 5277, Université de Toulouse, 14, Av. E.Belin, 31400 Toulouse, France \\ 4 Leibniz - Institut für Astrophysik Potsdam (AIP), An der Sternwarte 16, 14482 Potsdam, Germany \\ 5 Physics Department, Trieste University, via Tiepolo 11, 34143 Trieste, Italy \\ 6 INAF-Trieste Observatory, via Tiepolo 11, 34143 Trieste, Italy
}

Received 28 February 2012 / Accepted 8 April 2012

\begin{abstract}
Context. Recent studies of low- and intermediate-mass stars show that the evolution of the chemical elements in these stars is very different from that proposed by standard stellar models. Rotation-induced mixing modifies the internal chemical structure of main sequence stars, although its signatures are revealed only later in the evolution when the first dredge-up occurs. Thermohaline mixing is likely the dominating process that governs the photospheric composition of low-mass red giant branch stars and has been shown to drastically reduce the net ${ }^{3} \mathrm{He}$ production in these stars. The predictions of these new stellar models need to be tested against galaxy evolution. In particular, the resulting evolution of the light elements $\mathrm{D},{ }^{3} \mathrm{He}$ and ${ }^{4} \mathrm{He}$ should be compared with their primordial values inferred from the Wilkinson Microwave Anisotropy Probe data and with the abundances derived from observations of different Galactic regions.

Aims. We study the effects of thermohaline mixing and rotation-induced mixing on the evolution of the light elements in the Milky Way.

Methods. We compute Galactic evolutionary models including new yields from stellar models computed with thermohaline instability and rotation-induced mixing. We discuss the effects of these important physical processes acting in stars on the evolution of the light elements $\mathrm{D},{ }^{3} \mathrm{He}$, and ${ }^{4} \mathrm{He}$ in the Galaxy.

Results. Galactic chemical evolution models computed with stellar yields including thermohaline mixing and rotation fit better observations of ${ }^{3} \mathrm{He}$ and ${ }^{4} \mathrm{He}$ in the Galaxy than models computed with standard stellar yields.

Conclusions. The inclusion of thermohaline mixing in stellar models provides a solution to the long-standing " $3 \mathrm{He}$ problem" on a Galactic scale. Stellar models including rotation-induced mixing and thermohaline instability reproduce also the observations of D and ${ }^{4} \mathrm{He}$.
\end{abstract}

Key words. Galaxy: evolution - galaxies: abundances - galaxies: formation

\section{Introduction}

Understanding the evolution of the light elements deuterium (D), helium-3 $\left({ }^{3} \mathrm{He}\right)$, and helium-4 $\left({ }^{4} \mathrm{He}\right)$ hinges on the comprehension of several astrophysical processes and links together different branches of physics and cosmology. D, ${ }^{3} \mathrm{He}$, and ${ }^{4} \mathrm{He}$ are all synthesized in astrophysically relevant quantities during Big Bang nucleosynthesis (BBN; Peebles 1966; Wagoner et al. 1967). In the absence of any other realistic production channel (Epstein et al. 1976; Prodanović \& Fields 2003), the abundance of D in galaxies smoothly decreases in time as gas cycles through stars. ${ }^{3} \mathrm{He}$ and ${ }^{4} \mathrm{He}$, instead, have a more complex history, since they are both produced and destroyed in stars.

The sensitivity of the predicted BBN abundance of $\mathrm{D}$, $(\mathrm{D} / \mathrm{H})_{\mathrm{P}}$, to the baryon-to-photon ratio, $\eta_{\mathrm{B}}$, coupled with its straightforward galactic evolution, has long made this element be the "baryometer" of choice (Reeves et al. 1973). In the nineties, the controversial assessment of the primordial abundance of $\mathrm{D}$ from observations of high-redshift clouds (e.g. Songaila et al. 1994; Burles \& Tytler 1998), probing an almost unevolved medium, led researchers to resort to detailed Galactic chemical evolution (GCE) modelling in order to constrain the pre-Galactic D abundance and, hence, the value of $\eta_{\mathrm{B}}$. It was shown that any reasonable GCE model results in moderate local D astration, by less than a factor of three (Steigman \& Tosi 1992; Edmunds 1994; Galli et al. 1995; Dearborn et al. 1996; Prantzos 1996; Tosi et al. 1998; Chiappini et al. 2002). Assuming that the local present-day abundance of $\mathrm{D}$ is well known, this provided a stringent bound to $(\mathrm{D} / \mathrm{H})_{\mathrm{P}}$ and, hence, a test for $\mathrm{BBN}$ theories. Chemical evolution models able to reproduce the majority of the observational constraints for the Milky Way firmly ruled out the highest values of $(\mathrm{D} / \mathrm{H})_{\mathrm{P}}$ by Songaila et al. (1994). In particular, the values of $(\mathrm{D} / \mathrm{H})_{\mathrm{P}}$ and $\eta_{\mathrm{B}}$ suggested by Tosi (2000) and Chiappini et al. (2002) turned out to be in very good agreement with the ones determined from the analysis of the first Wilkinson Microwave Anisotropy Probe (WMAP) data (Bennett et al. 2003; Spergel et al. 2003).

Indeed, the first release of results from WMAP made us enter a new precision era for cosmology $-\eta_{\mathrm{B}}$ is now known with exquisite accuracy. Converging measurements of $\mathrm{D}$ abundances in remote gas clouds lead to $(\mathrm{D} / \mathrm{H})_{\mathrm{P}}=(2.8 \pm 0.2) \times 10^{-5}$ (Pettini et al. 2008), a value consistent, within the errors, with the 
Table 1. Abundances of deuterium, ${ }^{3} \mathrm{He}$, and ${ }^{4} \mathrm{He}$ at different epochs.

\begin{tabular}{lcccc}
\hline \hline Chemicals & Units & $\begin{array}{c}\text { SBBN+WMAP } \\
(t=0 \mathrm{Gyr})\end{array}$ & $\begin{array}{c}\text { Protosolar cloud } \\
(t=9.2 \mathrm{Gyr})\end{array}$ & $\begin{array}{c}\text { Local intestellar medium } \\
\text { (Present time })\end{array}$ \\
\hline $\mathrm{D}$ & $10^{5}(\mathrm{D} / \mathrm{H})$ & $2.49 \pm 0.17^{a}$ & $2.1 \pm 0.5^{c}$ & $2.31 \pm 0.24^{d}$ \\
& & $2.60_{-0.17}^{+0.19 b}$ & & $0.98 \pm 0.19^{e}$ \\
& & & $2.0 \pm 0.1^{f}$ \\
${ }^{3} \mathrm{He}$ & $10^{5}\left({ }^{3} \mathrm{He} / \mathrm{H}\right)$ & $1.00 \pm 0.07^{a}$ & $1.5 \pm 0.2^{c}$ & $2.4 \pm 0.7^{g}$ \\
& & $1.04 \pm 0.04^{b}$ & & \\
${ }^{4} \mathrm{He}$ & $\mathrm{Y}$ & $0.2486 \pm 0.0002^{a}$ & $0.2703^{h}$ & \\
& & $0.2479_{-0.0005}^{+0.0004 b}$ & & \\
\hline
\end{tabular}

References. ${ }^{(a)}$ Cyburt et al. (2008); ${ }^{(b)}$ Coc et al. (2004); ${ }^{(c)}$ Geiss \& Gloeckler (1998); ${ }^{(d)}$ Linsky et al. (2006); ${ }^{(e)}$ Hébrard et al. (2005); ${ }^{(f)}$ Prodanović et al. (2010); ${ }^{(g)}$ Gloeckler \& Geiss (1996); ${ }^{(h)}$ Asplund et al. (2009).

primordial $\mathrm{D}$ abundance predicted by the standard model of cosmology with parameters fixed by WMAP data (e.g. Cyburt et al. 2008; Coc et al. 2004, see Table 1). The remarkable homogeneity of $\mathrm{D}$ abundances at high redshifts, however, clashes with the unexpectedly large scatter in $\mathrm{D} / \mathrm{H}$ revealed by determinations of relatively local D abundances (Vidal-Madjar et al. 1998; Jenkins et al. 1999; Sonneborn et al. 2000; Hébrard et al. 2002; Oliveira \& Hébrard 2006). The observed dispersion can be reconciled with the predictions on D evolution from standard GCE models by taking into account two short-term, small-scale phenomena: $\mathrm{D}$ depletion onto dust grains and localized infall of primordial gas (Romano et al. 2006; Steigman et al. 2007; Romano 2010).

As far as ${ }^{3} \mathrm{He}$ is concerned, Iben (1967) and Truran \& Cameron (1971) first showed that large amounts of this element are produced by low-mass stars $\left(M \simeq 1-3 M_{\odot}\right)$ in the ashes of hydrogen burning by the p-p cycle on the main sequence. Problems arised soon, when Rood et al. (1976) incorporated these yields in models of GCE and came out with predicted ${ }^{3} \mathrm{He}$ abundances orders of magnitude higher than the observed ones in the Galaxy. Indeed, the nearly constancy of the ${ }^{3} \mathrm{He}$ abundance with both time and position within the Galaxy (e.g. Bania et al. 2002) rather implies a negligible production of this element in stars, at variance with predictions from standard stellar models. It was then advocated that some non-standard mechanisms are acting in a major fraction of low-mass stars, which prevents the fresh ${ }^{3} \mathrm{He}$ from surviving and being ejected in the interstellar medium (ISM; Rood et al. 1984). For instance, extra mixing was also invoked in order to explain other abundance anomalies like the carbon isotopic ratio in red giants (Charbonnel 1995; Hogan 1995; Charbonnel \& Do Nascimento 1998; see also Eggleton et al. 2006, 2008). The first ${ }^{3} \mathrm{He}$ stellar yields for low-mass stars computed with "ad hoc" extra-mixing (i.e., not related to a physical mechanism, see Sect. 2.2.2) became available with the work of Boothroyd \& Sackmann (1999). Galli et al. (1997); Palla et al. (2000); Chiappini et al. (2002) implemented those yields, accounting for the dependency of the helium-3 production/destruction on the stellar mass, metallicity and initial D abundances. It was shown that chemical evolution models which account for about $90 \%$ of low-mass stars undergoing extra-mixing led to a good agreement with the Proto Solar Cloud (PSC) observations as well as with the observed gradient along the disk.

The physical process responsible for the extra-mixing has possibly been recently identified. Charbonnel \& Zahn (2007b) have shown indeed that thermohaline instability, when modelled with a simple prescription based on linear stability analysis, leads to a drastic reduction of ${ }^{3} \mathrm{He}$ production in low-mass, low-metallicity red giant stars. However a couple of planetary nebulae, namely NGC 3242 and J320, have been found to behave classically (see Bania et al. 2010): slightly more massive than the Sun, they are currently returning fresh ${ }^{3} \mathrm{He}$ to the ISM, in agreement with standard predictions (Rood et al. 1992; Galli et al. 1997; Balser et al. 1999, 2006). To reconcile the ${ }^{3} \mathrm{He} / \mathrm{H}$ measurements in Galactic HII regions with the high values of ${ }^{3} \mathrm{He}$ in NGC 3242 and J320, Charbonnel \& Zahn (2007a) proposed that thermohaline mixing is inhibited by a fossil magnetic field in red giant branch (RGB) stars that are descendants of Ap stars. The percentage of such stars is about $2-10 \%$ of all A-type objects. This number agrees with the fact that about $4 \%$ of low-mass evolved stars (including NGC 3242) exhibit standard suface abundances as depicted by their carbon isotopic ratios (Charbonnel \& Do Nascimento 1998). Grids of ${ }^{3} \mathrm{He}$ yields from stellar models taking into account thermohaline instability, as well as rotational mixing, are presented in Lagarde et al. (2011). These are suitable for use in GCE studies such as the one presented here.

The scarce determinations of reliable ${ }^{4} \mathrm{He}$ abundances in the Galaxy (Balser et al. 2010; Peimbert et al. 2010), together with the steadiness of GCE model predictions on the evolution of ${ }^{4} \mathrm{He}$ when adopting different stellar yields (e.g. Chiappini et al. 2002; Romano et al. 2010), have given this element little attention in GCE studies.

In this paper, we deal with the evolution of $\mathrm{D},{ }^{3} \mathrm{He}$, and ${ }^{4} \mathrm{He}$ in the solar vicinity, as well as their distributions across the Galactic disc. The production of ${ }^{3} \mathrm{He}$ is strictly related to the destruction of $\mathrm{D}$ and the production of ${ }^{4} \mathrm{He}$. Therefore, these elements are considered all together. The main novelty of the present work is the new nucleosynthesis prescriptions for the synthesis of ${ }^{3} \mathrm{He}$ and ${ }^{4} \mathrm{He}$ in low-mass stars (masses below $6 M_{\odot}$ ). Here we present, for the first time, chemical evolution models for ${ }^{3} \mathrm{He}$ computed with stellar yields from non-standard stellar models that include both rotation-induced and thermohaline mixing (Charbonnel \& Zahn 2007a; Charbonnel \& Lagarde 2010; Lagarde et al. 2011). As we will show in the next sections, these yields bring chemical evolution model predictions into agreement with the Galactic ${ }^{3} \mathrm{He}$ data without the necessity of assuming "ad hoc" fractions of extra-mixing among low-mass stars.

The layout of the paper is as follows. In Sect. 2 we discuss the production/destruction channels of $\mathrm{D},{ }^{3} \mathrm{He}$, and ${ }^{4} \mathrm{He}$ in stars in light of new generation stellar models that take into account rotation-induced mixing and thermohaline instability. A comparison with stellar yields predictions from the literature is presented. In Sect. 3 we describe the adopted GCE model. In Sect. 4 

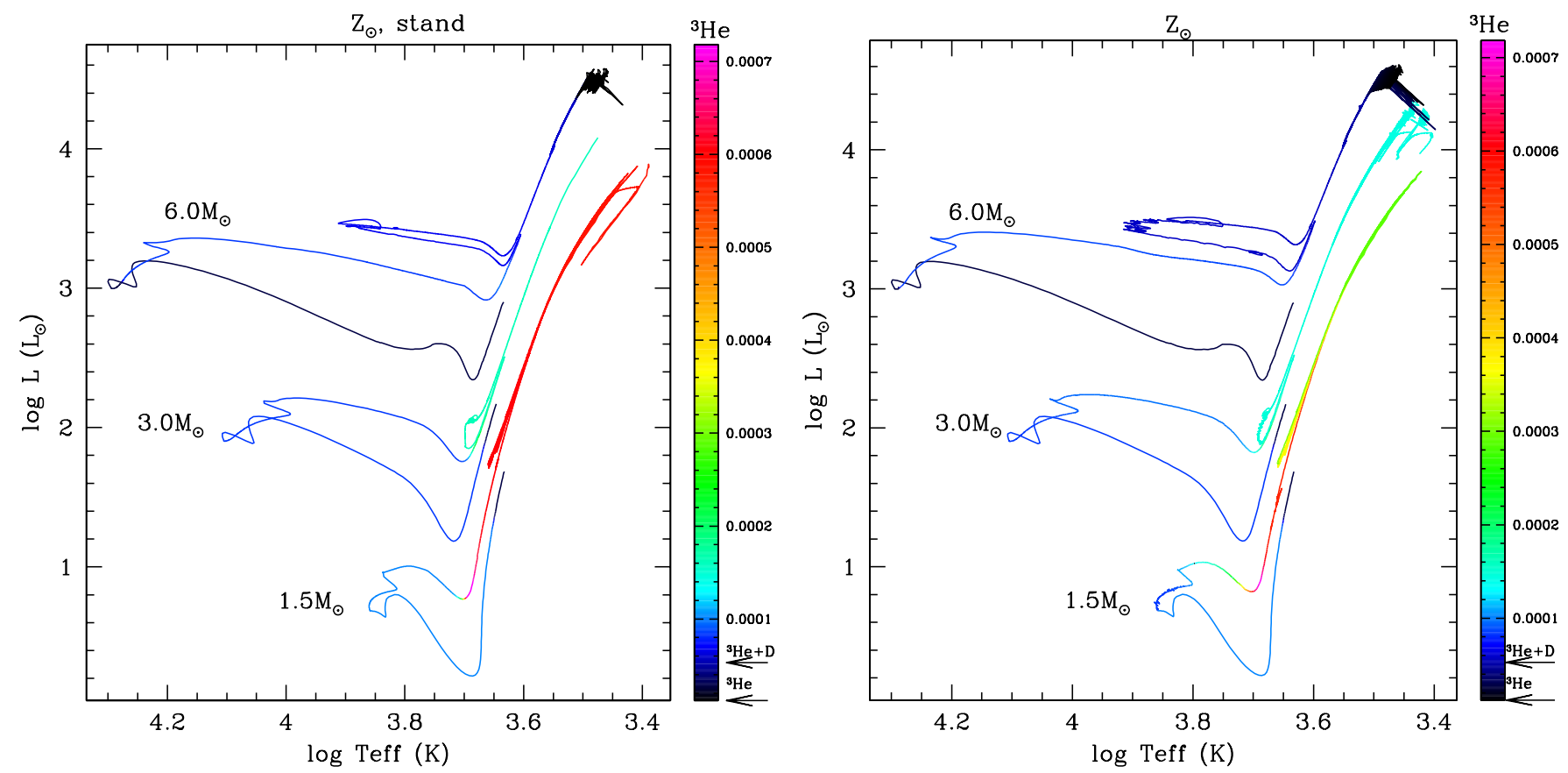

Fig. 1. Theoretical evolutionary tracks in the HR diagram for the $1.5 M_{\odot}, 3.0 M_{\odot}$, and $6.0 M_{\odot}$ models at solar metallicity following the standard prescription (left panel); and including rotation-induced mixing and thermohaline instability (right panel), from the pre-main sequence up to the end of the TP-AGB phase. Colours depict the mass fraction of ${ }^{3} \mathrm{He}$ at the stellar surface as indicated on the right, with the arrows showing the initial ${ }^{3} \mathrm{He}$ and ${ }^{3} \mathrm{He}+{ }^{2} \mathrm{H}$ content assumed at birth.

we discuss our results on the evolution of the light elements in the Galaxy. In particular, we try to put constraints on the uncertain parameters of stellar evolution through a comparison of the model predictions with the relevant data. We draw our conclusions in Sect. 5.

\section{Stellar nucleosynthesis of light elements from new generation models for low- and intermediate-mass stars}

In a series of papers (Charbonnel \& Lagarde 2010; Lagarde et al. 2011, 2012, hereinafter Papers I, II and III, respectively; see also Charbonnel \& Zahn 2007b,a), we discuss the impact of rotationinduced mixing and thermohaline instability on the structure, evolution, nucleosynthesis and yields, as well as on the asteroseismic and chemical properties of low- and intermediate-mass stars at various metallicities. A detailed description of the input physics of the models is given in Paper III. Rotation-induced mixing is treated using the complete formalism developed by Zahn (1992) and Maeder \& Zahn (1998) (see for more details, Papers I, II, III). In these stellar models, we consider that thermohaline instability develops as long thin fingers with the aspect ratio consistent with predictions by Ulrich (1972) and confirmed by the laboratory experiments (Krishnamurti 2003). We note that this value is higher than obtained by current 2D and 3D numerical simulations (Denissenkov 2010; Denissenkov \& Merryfield 2011; Rosenblum et al. 2011; Traxler et al. 2011). Before a final word on this discrepancy comes from future numerical simulations in realistic stellar conditions, we adopt an aspect ration (i.e., maximum length relative to their diameter) of 5, which nicely accounts for the observed chemical properties of red giant stars. These new generation stellar models account very nicely for main-sequence and RGB abundance patterns observed in field and open cluster stars over the mass and metallicity range covered, as shown in Paper I and in Charbonnel \& Zahn (2007b).
In this section we briefly summarize their characteristics as far as the nucleosynthesis of light elements is concerned.

\subsection{Deuterium}

Deuterium burns by proton-captures at low temperature during the pre-main sequence (Reeves et al. 1973). Therefore, it is totally destroyed in stellar interiors and the corresponding net yields are negative whatever the mass and metallicity of the star, as in the case of standard stellar models.

\subsection{Helium-3}

\subsubsection{Impact of rotation-induced mixing and thermohaline instability on model predictions}

Paper II describes in detail the behaviour of ${ }^{3} \mathrm{He}$ both in the standard case and in our models including thermohaline instability and rotation-induced mixing. Figure 1 shows the theoretical evolution of the ${ }^{3} \mathrm{He}$ surface abundance along the evolutionary tracks in the HR diagram for the models of 1.5, 3.0, and $6.0 M_{\odot}$ at solar metallicity that take into account these two processes. The colour coding in mass fraction is given on the right, with the initial values of ${ }^{3} \mathrm{He}$ and $\mathrm{D}+{ }^{3} \mathrm{He}$ indicated by the arrows. Evolution is shown from the pre-main sequence along the Hayashi track up to the end of the thermally-pulsing asymptotic giant branch (TP-AGB) phase. We see the changes in ${ }^{3} \mathrm{He}$ surface abundance due to D-burning on the pre-main sequence except in the $6.0 M_{\odot}$ model whose convective envelope withdraws very quickly at the beginning of that phase allowing to the preservation of pristine $\mathrm{D}$ in a very thin external layer. ${ }^{3} \mathrm{He}$ is then produced in low- and intermediate-mass stars during the main sequence through the pp-chains and subsequently dredgedup when the stars move towards the RGB. On the $1.5 M_{\odot}$ track one sees the effect of rotation-induced mixing that already brings fresh ${ }^{3} \mathrm{He}$ towards the stellar surface while the star is on the main 

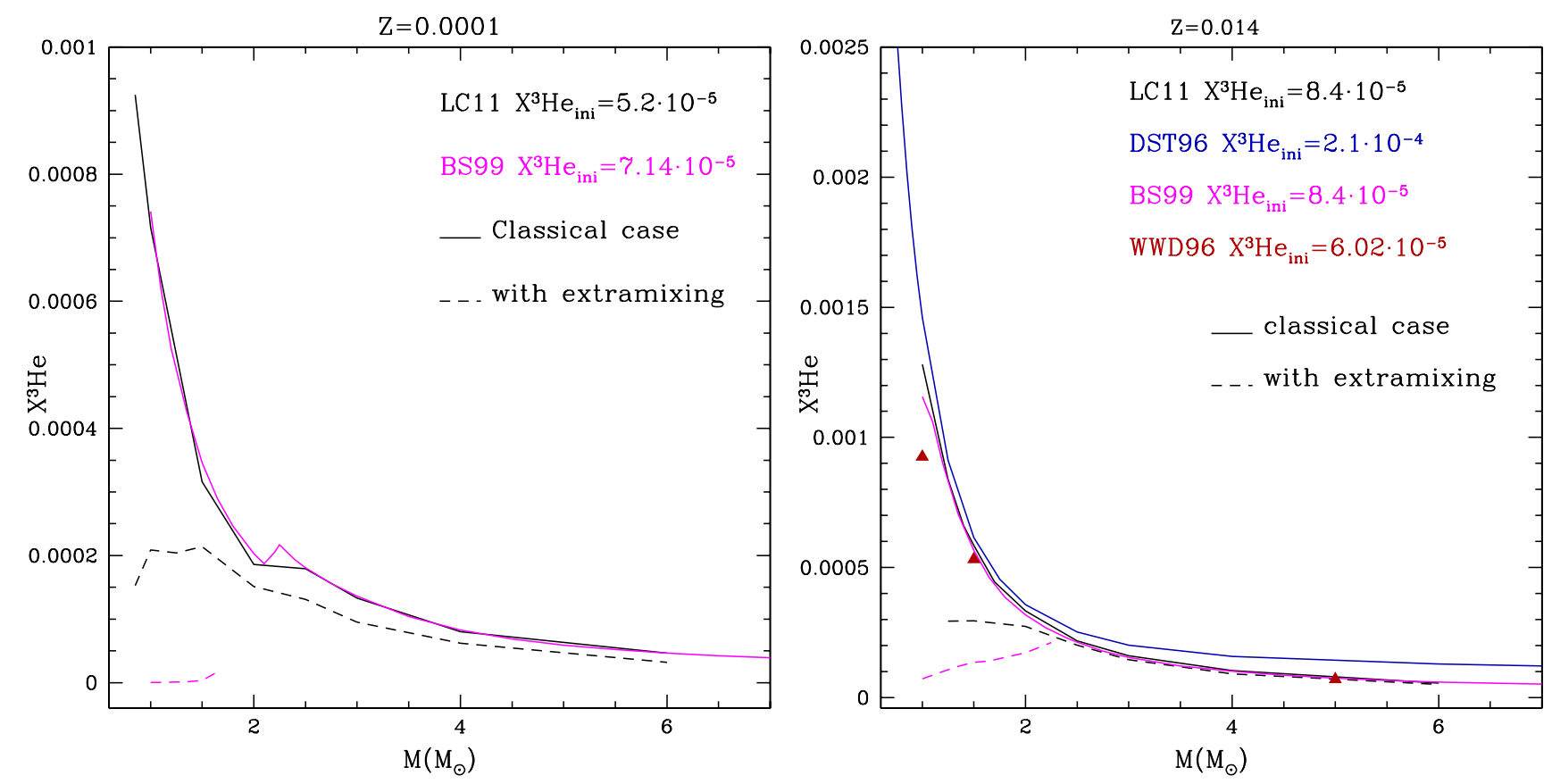

Fig. 2. Mass fraction of ${ }^{3} \mathrm{He}$ at the stellar surface at the end of the second dredge-up as a function of initial stellar mass in standard models (solid lines and triangles) and in models including various prescriptions for mixing in the radiative regions (dashed lines), at two metallicities $(Z=0.0001$ and $Z=0.014$; left and right panels, respectively). The predictions are from Dearborn et al. (1996, DST96, blue line), Boothroyd \& Sackmann (1999, BS99, at the RGB tip, magenta lines), Weiss et al. (1996, WWD96, red triangles), and Lagarde et al. (2011, LC11, black lines). The initial abundances of ${ }^{3} \mathrm{He}$ adopted at the zero age main sequence by the different authors are given on the top right corner of each panel.

sequence (on the contrary, in the standard case the surface abundance of ${ }^{3} \mathrm{He}$ remains constant on the main sequence and starts changing only at the very base of the RGB due to the first dredgeup). However, as discussed in Paper II rotation-induced mixing is found to lower the total ${ }^{3} \mathrm{He}$ production compared to the standard case over the whole mass and metallicity range scrutinized, and to decrease the upper mass limit at which stars destroy this element (see Fig. 5 in Paper II). Additionally, for low-mass stars $\left(M \leq 2-2.2 M_{\odot}\right)$ thermohaline mixing occurring during the RGB phase beyond the bump and on the TP-AGB leads to the destruction of part of the freshly produced ${ }^{3} \mathrm{He}$ while accounting for the observed surface abundance anomalies of other chemicals (i.e., lithium and nitrogen, as well as carbon isotopic ratio; see also Charbonnel \& Zahn 2007b); the associated decrease of surface ${ }^{3} \mathrm{He}$ is clearly seen for the $1.5 M_{\odot}$ model in Fig. 1 (right panel). Therefore, although low-mass stars remain net ${ }^{3} \mathrm{He}$ producers, their contribution to the Galactic evolution of this element is much lower than in the standard case. It was also shown in Paper II that thermohaline mixing leads to ${ }^{3} \mathrm{He}$ depletion during the TP-AGB phase for stars with masses up to $\sim 4 M_{\odot}$ (see the $3 M_{\odot}$ track in the figure). In more massive intermediate-mass stars, ${ }^{3} \mathrm{He}$ is further destroyed through hot-bottom burning on the TP-AGB. Finally, this figure shows that the lower the mass of the star, the higher the surface abundance of ${ }^{3} \mathrm{He}$ at the end of the TP-AGB. The global impact of rotation-induced mixing and thermohaline instability on the net ${ }^{3} \mathrm{He}$ yields from low- and intermediate-mass stars of various metallicities is summarized in Fig. 9 and in Tables 1 to 4 of Paper II (see also Fig. 2 discussed below).

\subsubsection{Comparison with other stellar models}

In Fig. 2 we show the mass fraction of ${ }^{3} \mathrm{He}$ at the end of the second dredge-up as a function of the initial stellar mass at two metallicities $(Z=0.0001$ and 0.014 ; left and right panels, respectively) from our models (black lines; see Paper II). A comparison is made with model predictions from the literature (coloured lines and filled triangles).

Our standard predictions (full lines) are in very good agreement with those from Dearborn et al. (1996), Boothroyd \& Sackmann (1999), and Weiss et al. (1996). For the reasons given in Sect. 2.2.1 they are higher than in the case including rotation-induced mixing and thermohaline instability (dashed black lines). Let us note that thermohaline mixing on the TPAGB leads to further decrease of the ${ }^{3} \mathrm{He}$ mass fraction at the stellar surface (reduction of 64,83 , and $19 \%$ in the $\left[1.25 M_{\odot}\right.$, $\left.Z_{\odot}\right],\left[0.85 M_{\odot}, Z=0.0001\right]$, and [2.0 $M_{\odot}, Z_{\odot}$ and $\left.Z=0.0001\right]$ models, respectively; see Paper II).

In Fig. 2 we also plot the predictions at the end of the second dredge-up for the models of Boothroyd \& Sackmann (1999, hereafter BS99) that include parametric mixing below the base of the convective envelope of RGB stars (the so-called "conveyor-belt" circulation and the associated "cool bottom processing", CBP; dashed magenta lines). In BS99 post-processing computations, the mixing is not related to any physical mechanism. Rather, the depth of the mixed zone is a free parameter that corresponds to the difference $\Delta \log T$ between the temperature at the base of the hydrogen-burning shell and that at the base of the assumed mixed zone. BS99 assumed that the value of $\Delta \log T$ remains constant along the RGB, and this free parameter was calibrated in order to reproduce the observed carbon isotopic ratio of clump stars in M 67. We note that the fixed $\Delta \log T$ value used by BS99 leads to shallower mixing just after the RGB bump than what we get when considering thermohaline instability as the physical mixing mechanism. As a consequence BS99 prescription leads to a slow and gradual theoretical decrease of the ${ }^{12} \mathrm{C} /{ }^{13} \mathrm{C}$ ratio up to the $\mathrm{RGB}$ tip; this behaviour disagrees with the sudden drop of this quantity that is observed 
after the RGB bump and that is well reproduced by our models that include thermohaline mixing (see Paper I and Charbonnel \& Zahn 2007b). However, due to the higher compactness of the hydrogen-burning shell when the stars reach higher luminosities on the RGB, the fixed $\Delta \log T$ value adopted by BS99 leads to deeper mixing close to the RGB tip. The second free parameter is the stream mass flow rate, $\dot{M}_{\mathrm{p}}$. Contrary to thermohaline mixing, for which the stream mass flow rate ${ }^{1}$ decreases along the RGB from $\sim 10^{-2.5}$ to $\sim 10^{-5} M_{\odot} \mathrm{yr}^{-1}$, BS99 assumed that the value of $\dot{M}_{\mathrm{p}}$ stays constant along the RGB $\left(\dot{M}_{\mathrm{p}}=10^{-4} M_{\odot} \mathrm{yr}^{-1}\right)$. This difference in the mixing efficiency at the end of the RGB explains the difference between BS99's models and ours on the final ${ }^{3} \mathrm{He}$ surface mass fraction. Another drawback of such models was also that a physical reason was missing to explain why only $7 \%$ of the low mass stars would produce ${ }^{3} \mathrm{He}$ following the standard predictions.

\subsection{Helium-4}

\subsubsection{Standard predictions}

Changes in the surface ${ }^{4} \mathrm{He}$ abundance result from the dredgeup episodes undergone by the stars along their evolution, that eventually lead to the partial engulfment of the ashes of centraland shell-H-burning by the convective envelopes. The efficiency of the successive dredge-up events depends both on stellar mass and metallicity (see e.g. Paper II), as depicted in Fig. 3 where we present our standard predictions (upper panels) for the surface abundance of ${ }^{4} \mathrm{He}$, adjusted to its initial value, in mass fraction, after the first and the second dredge-up (solid and dashed lines, respectively) as a function of the initial stellar mass, for our two extreme metallicities $\left(Z_{\odot}\right.$ and $\left.Z=0.0001\right)$. For solar metallicity, the surface abundance of ${ }^{4} \mathrm{He}$ increases during the first dredge-up over the whole mass range investigated, while for the lowest metallicity $\left(Z=10^{-4}\right)$ only stars with $M \leq 2.5 M_{\odot}$ are affected. On the other hand, the second dredge-up leads to an increase of the ${ }^{4} \mathrm{He}$ surface abundance only for the upper part of the considered mass range (i.e., for stars with masses higher than $\sim 2.5 M_{\odot}$ ). Finally, the third dredge-up, which occurs in stars with mass $\geq 6.0 M_{\odot}$ for $Z_{\odot}$ and $2.5 M_{\odot}$ for $Z=10^{-4}$ during the TP-AGB phase, allows to increase the ${ }^{4} \mathrm{He}$ abundance at the surface of these stars (not shown here). This increase is $\sim 1 \%$ for $\left[6.0 M_{\odot}, Z_{\odot}\right]$, and $\left[4.0 M_{\odot}, Z=0.0001\right]$ models $^{2}$.

\subsubsection{Impact of rotation-induced mixing and thermohaline instability on model predictions}

Thermohaline instability has no significant impact on the surface abundance of ${ }^{4} \mathrm{He}$ since it develops only in the upper wing

\footnotetext{
1 To translate the thermohaline diffusion coefficient of our models into a stream mass flow rate as used by BS99, we compute:

$\dot{M}_{\mathrm{p}}=\frac{4 \pi r^{2} \rho \mathcal{D}}{l_{\text {mix }}}$

with $r$ and $\rho$ the radius and the density at the base of the region where the thermohaline instability develops, $l_{\text {mix }}$ the size of this mixing zone, and $\mathcal{D}$ the mean diffusion coefficient along the mixing zone.

${ }^{2}$ Note that we did not include additional mechanisms to force the third dredge-up in our models, as required in the literature for reproducing the carbon-star luminosity function (e.g. Frogel et al. 1990; Costa \& Frogel 1996; Groenewegen 1998; Marigo et al. 1999). Hence, we do not attempt any comparison of the model predictions with available data for $\mathrm{C}$ and $\mathrm{N}$ isotopes. However this has no impact on the galactic chemical evolution of the light elements we focus on.
}

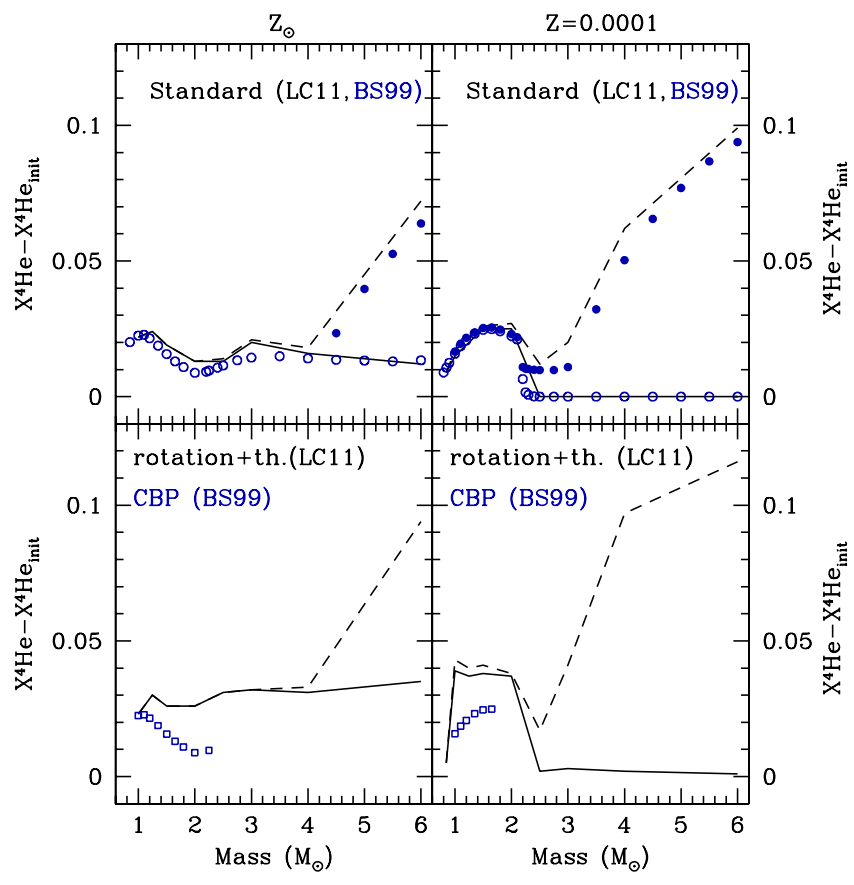

Fig. 3. Relative enrichment of the surface abundance of ${ }^{4} \mathrm{He}$ with respect to its initial value (in mass fraction) for Paper I models at the end of the first and second dredge-up (solid and dashed black lines, respectively) as a function of the initial stellar mass and for two metallicities ( $Z_{\odot}$ and $Z=0.0001$; left and right panels, respectively). Top panels: our standard predictions are compared with those of BS99 (blue circles; open and full at the end of the first and second dredge-up, respectively). Bottom panels: our models including both rotation-induced mixing and thermohaline instability are compared with the "cool-bottom processing" predictions by BS99 at the RGB tip (blue squares).

of the hydrogen-burning shell (see e.g. Paper I for more details $)^{3}$. However, rotation-induced mixing smoothes the internal abundance gradients compared to the standard case, leading freshly produced ${ }^{4} \mathrm{He}$ to diffuse outwards. This can be seen in Fig. 4 where we show the abundance profile of ${ }^{4} \mathrm{He}$ at the end of the main sequence for two stellar masses and metallicities in the standard and the rotating cases (black full and red dashed lines, respectively; the vertical bars indicate the depth reached by the convective envelope at its maximum extent during the first dredge-up). This results in stronger ${ }^{4} \mathrm{He}$ abundance variations at the end of the first dredge-up when rotation-induced mixing is accounted for, as seen in Fig. 3 (bottom panels). For the same reasons, the effect of the second dredge-up is also strengthened in the rotating models. Overall, the impact of rotation increases with decreasing metallicity (see Paper I, and references therein).

Consequently, the yields of ${ }^{4} \mathrm{He}$ are higher in the models including rotation-induced mixing than in the standard case (see Tables 3 to 6). And at the same time, the fraction of $\mathrm{H}$ is decreased.

Figure 5 summarizes all the effects described above on the surface abundance of ${ }^{4} \mathrm{He}$ along the evolution of standard and rotating stars with different initial masses at solar metallicity. The tracks are shown from the zero age main sequence up to the end of the TP-AGB, with the colour coding given on the right scale.

3 Thermohaline mixing leads to an increase of the surface abundance of ${ }^{4} \mathrm{He}$ during the RGB by $\sim 0.1 \%$ in the $1.25 M_{\odot}$ models independently of the stellar metallicity. 


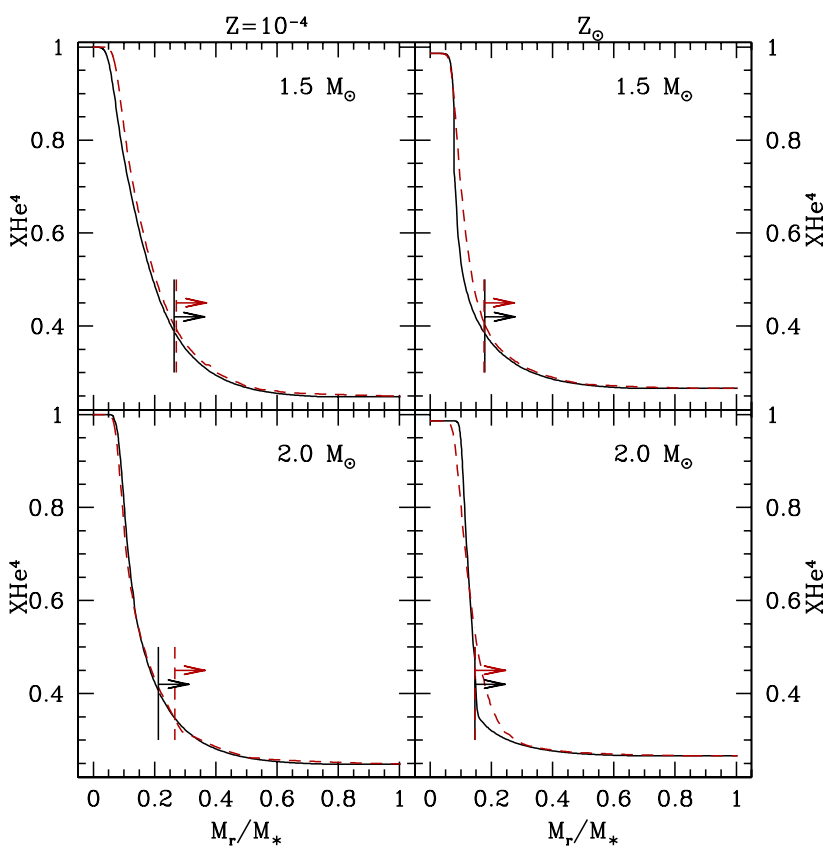

Fig. 4. Abundance profile of ${ }^{4} \mathrm{He}$ at the end of the main sequence for the $1.5 M_{\odot}$ and $2.0 M_{\odot}$ models at two metallicities $(Z=0.0001$ and $Z=0.014$, left and right panel, respectively) in the standard case (solid black line), and when including the effects of rotation (red dashed line). Models are from Paper II. The maximum depth reached by the convective envelope during the first dredge-up is shown with the vertical bars and arrows.

\subsubsection{Comparison with other stellar models}

In Fig. 3 we compare our predictions with those of Boothroyd \& Sackmann (1999). We note a very good agreement as far as the standard models are concerned (upper panels). On the other hand, neither the thermohaline instability nor BS99 "cool bottom processing" do affect the ${ }^{4} \mathrm{He}$ surface abundances. The differences that appear in the lower panels result essentially from the effects of rotation-induced mixing.

\section{Chemical evolution model}

\subsection{Basic assumptions}

The adopted model for the chemical evolution of the Galaxy assumes that the Milky Way forms out of two main accretion episodes almost completely disentangled (Chiappini et al. 1997, 2001). During the first one, the primordial gas collapses very quickly and forms the spheroidal components, halo and bulge. During the second one, the thin-disc forms, mainly by accretion of matter of primordial chemical composition. The disc is built-up in the framework of the inside-out scenario of Galaxy formation, which ensures the formation of abundance gradients along the disc (Larson 1976; Matteucci \& Francois 1989). The Galactic disc is approximated by several independent rings, $2 \mathrm{kpc}$ wide, without exchange of matter between them.

\subsection{Nucleosynthesis prescriptions}

The nucleosynthesis prescriptions for metals (elements heavier than ${ }^{4} \mathrm{He}$ ) are from Paper II for low- and intermediate-mass stars $\left(M \leq 6 M_{\odot}\right)$. As for massive stars and Type Ia supernovae, we adopt the same nucleosynthesis prescriptions as in Romano et al. (2010), their model 6, namely:

- yields for core-collapse supernovae are taken from Kobayashi et al. (2006), except for carbon, nitrogen and
Table 2. Description of different models computed with yields from Lagarde et al. (2011) for stars below $6 M_{\odot}$.

\begin{tabular}{lcc}
\hline \hline Models & \multicolumn{2}{c}{ Stellar mass } \\
& $M \leq 2.5 M_{\odot}$ & $M>2.5 M_{\odot}$ \\
\hline A & $100 \%$ standard & $100 \%$ standard \\
B & $96 \%$ th. $+\operatorname{rot}^{1}$ & $100 \%$ th. + rot \\
& $4 \%$ standard & \\
C & $100 \%$ th. + rot & $100 \%$ th. + rot \\
\hline
\end{tabular}

Notes. ${ }^{(1)}$ th. + rot means that we use yields including thermohaline instability and rotation induced mixing.

oxygen, for which the adopted yields are from Meynet \& Maeder (2002); Hirschi et al. (2005); Hirschi (2007); Ekström et al. (2008);

- yields for Type Ia supernovae are from Iwamoto et al. (1999).

As far as the nucleosynthesis prescriptions for $\mathrm{D},{ }^{3} \mathrm{He}$ and ${ }^{4} \mathrm{He}$ are concerned, we use standard prescriptions from Dearborn et al. (1996) in the 6 to $100 M_{\odot}$ mass range for D and ${ }^{3} \mathrm{He}$ and yields by Meynet \& Maeder (2002); Hirschi et al. (2005); Hirschi (2007); Ekström et al. (2008) for ${ }^{4} \mathrm{He}$. For low- and intermediate-mass stars $\left(M \leq 6 M_{\odot}\right)$ we use the yields from Papers II and III $^{4}$ and we compute three different models with the following assumptions (summarized in Table 2):

- In Model A we consider the standard yields for all stars with masses below $6.0 M_{\odot}$.

- In Model B we consider the yields including thermohaline instability and rotation-induced mixing for $100 \%$ of the intermediate-mass stars $\left(M>2.5 M_{\odot}\right)$ and $96 \%$ of the lowmass stars $\left(M \leq 2.5 M_{\odot}\right)$. The remaining $4 \%$ of the low-mass stars are assumed to release the yields as predicted by the standard theory. This allows us to account for the fact that some rare planetary nebulae (J320, NGC 3242) exhibit ${ }^{3} \mathrm{He}$ as predicted by the standard models (see e.g. Balser et al. 2007, and references therein).

- In Model C we assume the yields including thermohaline instability and rotation-induced mixing for all the low- and intermediate-mass stars. Obviously the outcome of Models B and $\mathrm{C}$ are expected to be very similar.

Linear interpolations in mass and metallicity are used to fill the gaps in the computed grids of yields (see Romano et al. 2010, for a discussion of the potential spurious effects introduced by this procedure).

\section{Evolution of the light elements in the Milky Way}

In the following sections we discuss the evolution of the light elements in the Galaxy within the framework described in Sect. 3 when taking into account the yields from our non-standard stellar models, and compare the predictions with different observations. The relevant data are presented in Table 1.

\subsection{Evolution of deuterium}

In this paper we assume $(\mathrm{D} / \mathrm{H})_{\mathrm{P}}=2.6 \times 10^{-5}$ for consistency with the initial value adopted by the stellar models. However, we note that our GCE model gives a better fit to the data when

\footnotetext{
${ }^{4}{ }^{3} \mathrm{He}$ stellar yields are presented in Tables 1 to 4 of Paper II. ${ }^{4} \mathrm{He}$ stellar yields are shown in Tables 3 to 6 .
} 
N. Lagarde et al.: Evolution of light elements in the Galaxy: D, ${ }^{3} \mathrm{He}$ and ${ }^{4} \mathrm{He}$
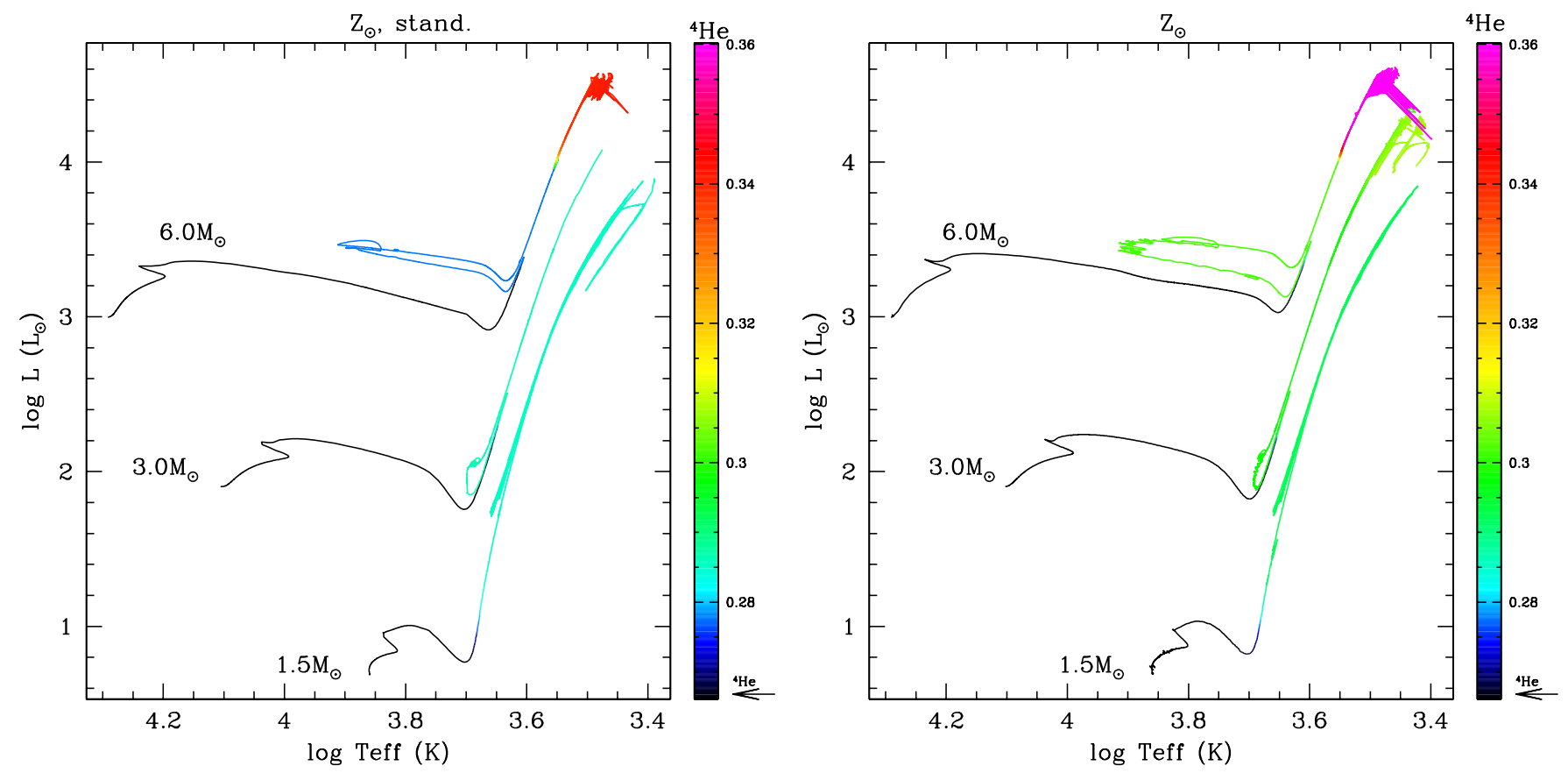

Fig. 5. Same as Fig. 1 for ${ }^{4} \mathrm{He}$ from the zero age main sequence to the end of the TP-AGB.
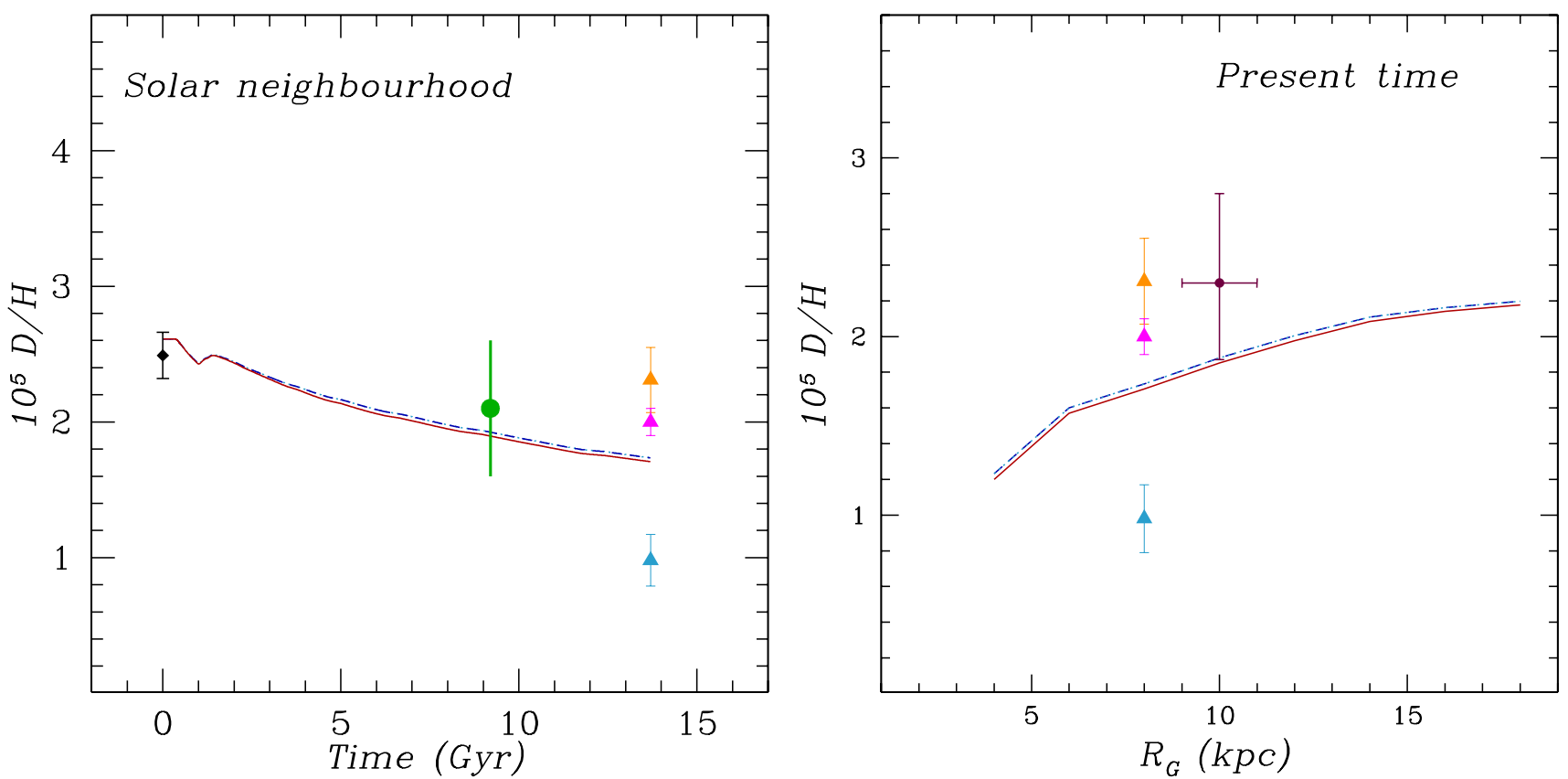

Fig. 6. Evolution of $\mathrm{D} / \mathrm{H}$ in the solar neighborhood (left panel) and distribution of $\mathrm{D} / \mathrm{H}$ along the Galactic disc at the present time (right panel). The predictions of Models A and B are shown (red solid and dashed blue lines, respectively). At $t=0$ Gyr, we plot the WMAP value (filled black diamond). The PSC data by Geiss \& Gloeckler (1998) are shown (filled green circle). The local interstellar medium (LISM) data are from Linsky et al. (2006), Hébrard et al. (2005) and Prodanović et al. (2010) (filled orange, light blue, and magenta triangles, respectively). The data for the outer disc $\left(R_{\mathrm{G}}=10 \mathrm{kpc}\right)$ are from Rogers et al. (2005, filled bordeaux circle).

a value of $(\mathrm{D} / \mathrm{H})_{\mathrm{P}}=2.8 \times 10^{-5}$ as observed in quasar spectra (Pettini et al. 2008) is adopted instead (see Fig. 3 of Romano 2010).

Figure 6 (left panel) shows the data for deuterium from the Big Bang (WMAP value, filled black diamond) to the present day (local interstellar medium, LISM, filled triangles). The determinations of primordial and PSC (filled green circle, Geiss \& Gloeckler 1998, according with Geiss \& Reeves 1972, 1981) deuterium abundances underline a small depletion from the Big Bang ( $t=0 \mathrm{Gyr})$ to the solar-system formation $(t=9.2 \mathrm{Gyr})$.
Analyses of Far Ultraviolet Spectroscopic Explorer (FUSE) observations have allowed measurements of $\mathrm{D} / \mathrm{H}$ in the LISM for many lines of sight. These observations have revealed a large variation of the local $\mathrm{D}$ abundances, which complicates the interpretation in the context of standard GCE models (Linsky et al. 2006; Linsky 2010). Hébrard et al. (2005) and Linsky et al. (2006) proposed that either the lowest $\left(\mathrm{D} / \mathrm{H}=0.98 \times 10^{-5}\right)$ or the highest $\left(\mathrm{D} / \mathrm{H}=2.31 \times 10^{-5}\right)$ observed value is indicative of the true LISM value reflecting the process of $\mathrm{D}$ astration through successive stellar generations during the whole Galaxy's 
Table 3. Model results for metallicity of $Z=0.0001$.

\begin{tabular}{lcccc}
\hline \hline $\begin{array}{l}M \\
\left(M_{\odot}\right)\end{array}$ & & $V_{\text {ZAMS }} / V_{\text {crit }}{ }^{1}$ & $\begin{array}{c}V_{\text {ZAMS }}{ }^{1} \\
\left(\mathrm{~km} \mathrm{~s}^{-1}\right)\end{array}$ & $\begin{array}{c}\text { Yield }{ }^{4} \mathrm{He}^{2} \\
\left(M_{\odot}\right)\end{array}$ \\
\hline 0.85 & stand. & - & - & $5.22 \times 10^{-03}$ \\
& th. +rot. & 0.45 & 115 & $1.29 \times 10^{-02}$ \\
1.0 & stand. & - & - & $9.41 \times 10^{-03}$ \\
& th. + rot. & 0.45 & 116 & $2.42 \times 10^{-02}$ \\
1.25 & th. +rot. & 0.45 & 125 & $2.98 \times 10^{-02}$ \\
1.5 & stand. & - & - & $2.44 \times 10^{-02}$ \\
& th. +rot. & 0.45 & 134 & $3.74 \times 10^{-02}$ \\
2.0 & stand. & - & - & $3.38 \times 10^{-02}$ \\
& th. +rot. & 0.45 & 150 & $4.89 \times 10^{-02}$ \\
2.5 & stand. & - & - & $1.90 \times 10^{-02}$ \\
& th. +rot. & 0.45 & 162 & $2.77 \times 10^{-02}$ \\
3.0 & stand. & - & - & $3.99 \times 10^{-02}$ \\
& th. +rot. & 0.45 & 170 & $8.21 \times 10^{-02}$ \\
4.0 & stand. & - & - & $1.67 \times 10^{-02}$ \\
& th. +rot. & 0.45 & 152 & $2.75 \times 10^{-02}$ \\
6.0 & stand. & - & - & $4.09 \times 10^{-02}$ \\
& th. +rot. & 0.45 & 175 & $4.79 \times 10^{-02}$ \\
\hline
\end{tabular}

Notes. Each row contains entries for different assumptions: standard (without thermohaline or rotation-induced mixing); thermohaline mixing only; thermohaline and rotation-induced mixing. ${ }^{(1)}$ The initial rotation on the ZAMS. ${ }^{(2)}$ Yields of ${ }^{4} \mathrm{He}$.

Table 4. Same as Table 3 for $Z=0.002$.

\begin{tabular}{lcccc}
\hline \hline $\begin{array}{l}M \\
\left(M_{\odot}\right)\end{array}$ & & $V_{\text {ZAMS }} / V_{\text {crit }}$ & $\begin{array}{c}V_{\text {ZAMS }} \\
\left(\mathrm{km} \mathrm{s}^{-1}\right)\end{array}$ & $\begin{array}{c}\text { Yield }{ }^{4} \mathrm{He} \\
\left(M_{\odot}\right)\end{array}$ \\
\hline 0.85 & stand. & - & - & $7.47 \times 10^{-03}$ \\
& th. + rot. & 0.45 & 114 & $1.61 \times 10^{-02}$ \\
1.0 & stand. & - & - & $1.18 \times 10^{-02}$ \\
& th. + rot. & 0.45 & 112 & $2.68 \times 10^{-02}$ \\
1.25 & th. + rot. & 0.45 & 115 & $2.90 \times 10^{-02}$ \\
1.5 & stand. & - & - & $3.13 \times 10^{-02}$ \\
& th. + rot. & 0.45 & 123 & $4.20 \times 10^{-02}$ \\
2.0 & stand. & - & - & $2.13 \times 10^{-02}$ \\
& th. + rot. & 0.45 & 137 & $4.71 \times 10^{-02}$ \\
2.5 & stand. & - & - & $2.15 \times 10^{-02}$ \\
& th. + rot. & 0.45 & 146 & $5.84 \times 10^{-02}$ \\
3.0 & stand. & - & - & $9.84 \times 10^{-03}$ \\
& th. + rot. & 0.45 & 153 & $5.76 \times 10^{-02}$ \\
4.0 & stand. & - & - & $1.28 \times 10^{-01}$ \\
& th. + rot. & 0.45 & 163 & $2.28 \times 10^{-01}$ \\
6.0 & stand. & - & - & $3.92 \times 10^{-01}$ \\
& th. + rot. & 0.45 & 170 & $4.30 \times 10^{-01}$ \\
\hline
\end{tabular}

evolution. Hébrard et al. (2005) suggest a value of the true local deuterium abundance lower than the one measured in the local bubble. On the other hand, Linsky et al. (2006) give a lower bound to the true local deuterium abundance very close to the primordial abundance, pointing to a deuterium astration factor smaller than predicted by standard GCE models. More recently, Prodanović et al. (2010) have applyed a statistical Bayesian method to determine the true local $\mathrm{D}$ abundance. They propose a value very close to the $\mathrm{D}$ abundance at the time of the formation of the Sun (see Table 1). GCE models that fulfil all the major observational constraints available for the solar neighbourhood and for the Milky Way disc can explain the LISM D abundance
Table 5. Same as Table 3 for $Z=0.004$.

\begin{tabular}{lcccc}
\hline \hline $\begin{array}{l}M \\
\left(M_{\odot}\right)\end{array}$ & & $V_{\text {ZAMS }} / V_{\text {crit }}$ & $\begin{array}{c}V_{\text {ZAMS }} \\
\left(\mathrm{km} \mathrm{s}^{-1}\right)\end{array}$ & $\begin{array}{c}\text { Yield }{ }^{4} \mathrm{He} \\
\left(M_{\odot}\right)\end{array}$ \\
\hline 1.0 & stand. & - & - & $1.24 \times 10^{-02}$ \\
& th. + rot. & 0.45 & 112 & $2 \times 10 \times 10^{-02}$ \\
1.25 & th. + rot. & 0.45 & 111 & $2.84 \times 10^{-02}$ \\
1.5 & stand. & - & - & $1.95 \times 10^{-02}$ \\
& th. + rot. & 0.45 & 119 & $2.83 \times 10^{-02}$ \\
2.0 & stand. & - & - & $1.90 \times 10^{-02}$ \\
& th. + rot. & 0.45 & 123 & $3.33 \times 10^{-02}$ \\
2.5 & stand. & - & - & $2.94 \times 10^{-02}$ \\
& th. + rot. & 0.45 & 141 & $5.62 \times 10^{-02}$ \\
3.0 & stand. & - & - & $2.03 \times 10^{-02}$ \\
& th. + rot. & 0.45 & 147 & $6.75 \times 10^{-02}$ \\
4.0 & stand. & - & - & $7.83 \times 10^{-02}$ \\
& th. + rot. & 0.45 & 147 & $1.93 \times 10^{-01}$ \\
6.0 & stand. & - & - & $3.49 \times 10^{-01}$ \\
& th. + rot. & 0.45 & 167 & $4.14 \times 10^{-01}$ \\
\hline & & & &
\end{tabular}

Table 6. Same as Table 3 for $Z=0.014$.

\begin{tabular}{lcccc}
\hline \hline $\begin{array}{l}M \\
\left(M_{\odot}\right)\end{array}$ & & $V_{\text {ZAMS }} / V_{\text {crit }}$ & $\begin{array}{c}V_{\text {ZAMS }} \\
\left(\mathrm{km} \mathrm{s}^{-1}\right)\end{array}$ & $\begin{array}{c}\text { Yield }{ }^{4} \mathrm{He} \\
\left(M_{\odot}\right)\end{array}$ \\
\hline 1.0 & stand. & - & - & $1.29 \times 10^{-02}$ \\
1.25 & th. + rot. & 0.45 & 110 & $2.27 \times 10^{-02}$ \\
1.5 & stand. & - & - & $2.79 \times 10^{-02}$ \\
& th. + rot. & 0.45 & 110 & $2.45 \times 10^{-02}$ \\
2.0 & stand. & - & - & $1.65 \times 10^{-02}$ \\
& th. + rot. & 0.45 & 110 & $3.36 \times 10^{-02}$ \\
2.5 & stand. & - & - & $2.35 \times 10^{-02}$ \\
& th. + rot. & 0.45 & 130 & $5.36 \times 10^{-02}$ \\
3.0 & stand. & - & - & $4.08 \times 10^{-02}$ \\
& th. +rot. & 0.45 & 136 & $8.09 \times 10^{-02}$ \\
4.0 & stand. & - & - & $4.80 \times 10^{-02}$ \\
& th. + rot. & 0.45 & 144 & $8.98 \times 10^{-02}$ \\
6.0 & stand. & - & - & $3.07 \times 10^{-01}$ \\
& th. + rot. & 0.45 & 156 & $3.84 \times 10^{-01}$ \\
\hline
\end{tabular}

suggested by Prodanović et al. (2010) as a result of D astration during Galactic evolution; lower and higher values can only be explained as due to small-scale, transient phenomena, such as $\mathrm{D}$ depletion on to dust grains and localized infall of gas of primordial chemical composition (Romano et al. 2006; Steigman et al. 2007; Romano 2010, and references therein).

Our predictions for the evolution of deuterium with time in the solar neighborhood and for the present deuterium abundance profile along the Milky Way disc are shown in Fig. 6 (left and right panels, respectively). The very modest shift between the predictions of Models A and B is due to the slight difference in the $\mathrm{H}$ yields when stellar rotation is accounted for compared to the standard case. Since pristine deuterium burns entirely in stars independently of their mass and metallicity, the predicted trends depend only on the total astration assumed in the GCE model. The present predictions fit well the PSC deuterium data and favour the local abundance of deuterium suggested by Prodanović et al. (2010). 
N. Lagarde et al.: Evolution of light elements in the Galaxy: D, ${ }^{3} \mathrm{He}$ and ${ }^{4} \mathrm{He}$
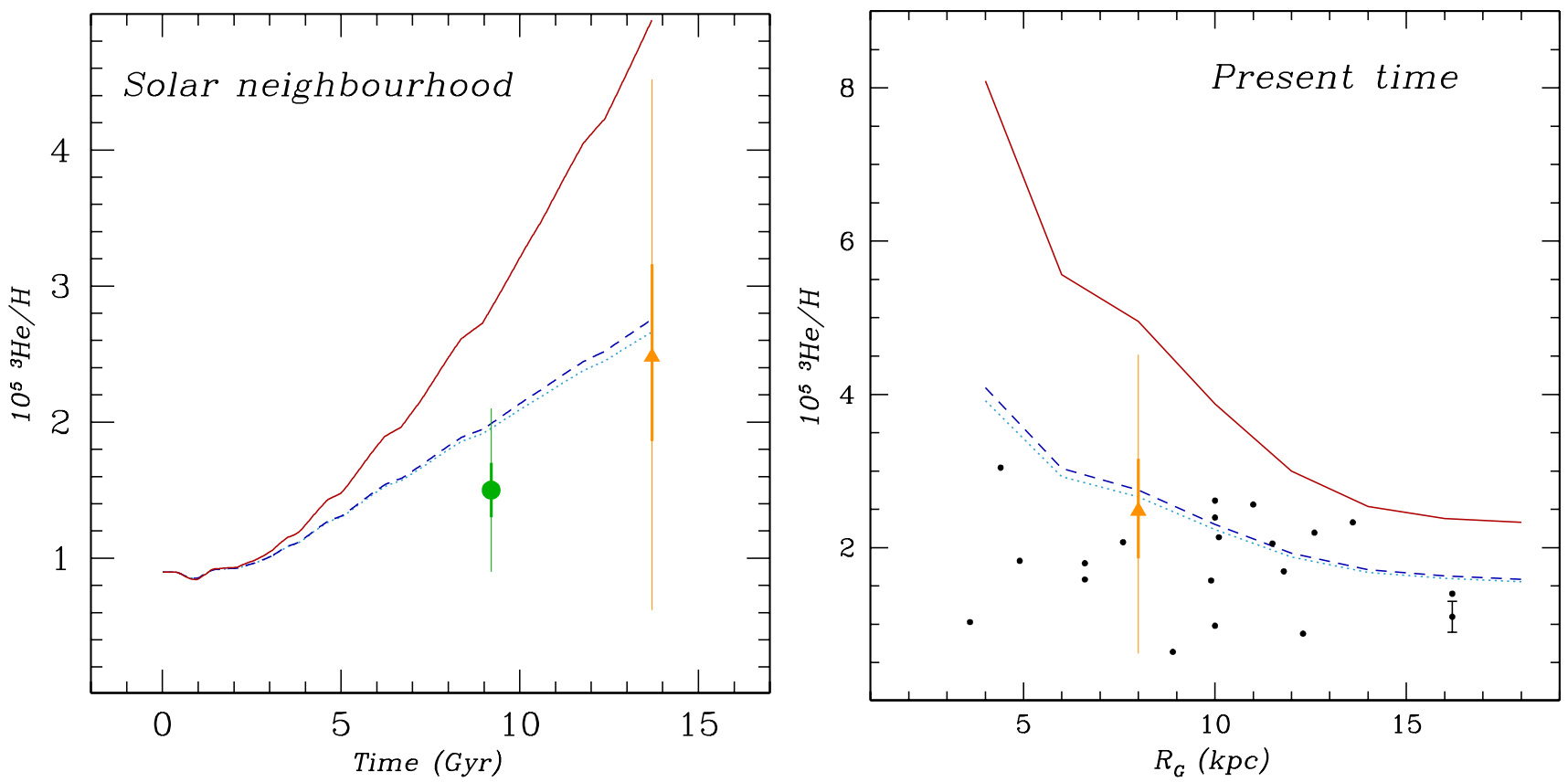

Fig. 7. Left panel: evolution of ${ }^{3} \mathrm{He} / \mathrm{H}$ with time in the solar neighborhood. Data for the PSC (green filled circle) and local interstellar medium (LISM, orange filled triangle) are from Geiss \& Gloeckler (1998) and Gloeckler \& Geiss (1996), respectively. 1- $\sigma$ and 3- $\sigma$ error bars are shown with thick and thin lines, respectively. Right panel: radial distribution of ${ }^{3} \mathrm{He} / \mathrm{H}$ at the present time. The dots are HII regions data from Bania et al. (2002) (error bars are shown only for S209; see text for discussion). The triangle at $R_{\mathrm{G}}=8 \mathrm{kpc}$ represents LISM data from Gloeckler \& Geiss (1996). The predictions from Models A, B and C are shown in both panels by the red full, blue dashed and cyan dotted lines respectively.

\subsection{Evolution of ${ }^{3} \mathrm{He}$}

Figure 7 (left panel) shows the evolution of ${ }^{3} \mathrm{He} / \mathrm{H}$ in the solar neighborhood as predicted by Models A, B, and C (see Sect. 3.2). As already discussed in the literature (see Sect. 1), standard stellar models strongly overestimate the production of ${ }^{3}$ He by low-mass stars.

According to standard predictions, ${ }^{3} \mathrm{He}$ is produced on the main sequence during the core hydrogen burning and it is not destroyed during later phases. Consequently, when adopting standard predictions ${ }^{3} \mathrm{He}$ is overproduced in the course of Galactic evolution (Model A, red solid line). In low-mass stellar models including thermohaline mixing, ${ }^{3} \mathrm{He}$ is destroyed from the bump luminosity on the RGB during shell hydrogen burning and during the early AGB. When low-mass stars are assumed to experience this extra-mixing, GCE models do not overproduce ${ }^{3} \mathrm{He}$ (Fig. 7, Model C, cyan dotted line). Model B (dashed blue line) shows the effect of inhibiting the thermohaline mixing in $4 \%$ of low-mass stars (see Sect. 3.2), that thus follow the standard prescriptions. As can be seen in Fig. 7, negligible differences are found between Model B and Model C predictions. The PSC and local ${ }^{3} \mathrm{He}$ abundances (Geiss \& Gloeckler 1998; Gloeckler \& Geiss 1996) are reproduced by the models (at 3- and 1-sigma level, respectively).

The present-day ${ }^{3} \mathrm{He}$ abundance distribution along the Galactic disk is shown in Fig. 7 (right panel). Model A, adopting standard ${ }^{3} \mathrm{He}$ yields for low-mass stars, predicts too much ${ }^{3} \mathrm{He}$ at all Galactocentric distances. In the inner regions, where the star formation was stronger, the contribution of low- and intermediate-mass stars to the ${ }^{3} \mathrm{He}$ enrichment of the ISM is more important. As a consequence, Model A predicts a large negative gradient of ${ }^{3} \mathrm{He} / \mathrm{H}$. Although the contribution of lowmass stars in the inner regions is significantly reduced in Model B, it still predicts a negative gradient. In Fig. 7, we compare the predictions of Models A and B with observations of
${ }^{3} \mathrm{He}$ in HII regions from Bania et al. (2002) and with the local ${ }^{3} \mathrm{He}$ abundance from Gloeckler \& Geiss (1996). Contrary to what is predicted by classical theory, the observations in HII regions show a gradient close to zero. Bania et al. (2002) derived meaningful error estimates only for one source [S209: $\left.{ }^{3} \mathrm{He} / \mathrm{H}=(1.1 \pm 0.2) \times 10^{-5}\right)$ and pointed our the riskiness of basing one's conclusion on only one object. In order to better constrain both stellar and galactic evolution studies, it would be very useful if future work could provide a sound estimate of the errors associated to ${ }^{3} \mathrm{He}$ determinations across the Galactic disk. Nevertheless, we can conclude that thermohaline and rotating mixing are required to fit currently available measurements of ${ }^{3} \mathrm{He}$ in Galactic HII regions. In addition, the ${ }^{3} \mathrm{He}$ gradient is $\mathrm{d} \log \left({ }^{3} \mathrm{He} / \mathrm{H}\right) / \mathrm{d} R_{\mathrm{G}} \sim-0.04$ and $-0.028 \mathrm{dex} / \mathrm{kpc}$ for Models A and $\mathrm{B}$, respectively, consistent with that predicted by Chiappini et al. (2002), $-0.04<\mathrm{d} \log \left({ }^{3} \mathrm{He} / \mathrm{H}\right) / \mathrm{d} R_{\mathrm{G}}<-0.03 \mathrm{dex} / \mathrm{kpc}$.

Tosi (2000) showed that GCE models including the CBP (Boothroyd \& Sackmann 1999) also fit the relevant Galactic ${ }^{3} \mathrm{He}$ data, provided the primordial abundance of $\mathrm{D}$ is sufficiently low (see also Chiappini et al. 2002; Romano et al. 2003). However, CBP destructs ${ }^{3} \mathrm{He}$ too quickly and is not a physical mechanism (see Sect. 2.2). Therefore, it does not provide a reliable physical explanation of the ${ }^{3} \mathrm{He}$ observations in HII regions.

\subsection{Evolution of ${ }^{4} \mathrm{He}$}

Figure 8 shows the temporal (left panel) and the spatial (right panel) evolution of the helium isotopic ratio $\left({ }^{3} \mathrm{He} /{ }^{4} \mathrm{He}\right)$ in the solar neighborhood and along the Galactic disk at the present time, respectively. Only the predictions of Models A and B are shown in the right panel. The PSC and LISM data are from Geiss \& Gloeckler (1998) and Gloeckler \& Geiss (1996), respectively. The standard theory predicts a significant evolution of the helium isotopic ratio in the Galaxy, and a strong gradient across the Galactic disc. When the thermohaline mixing - which destroys 

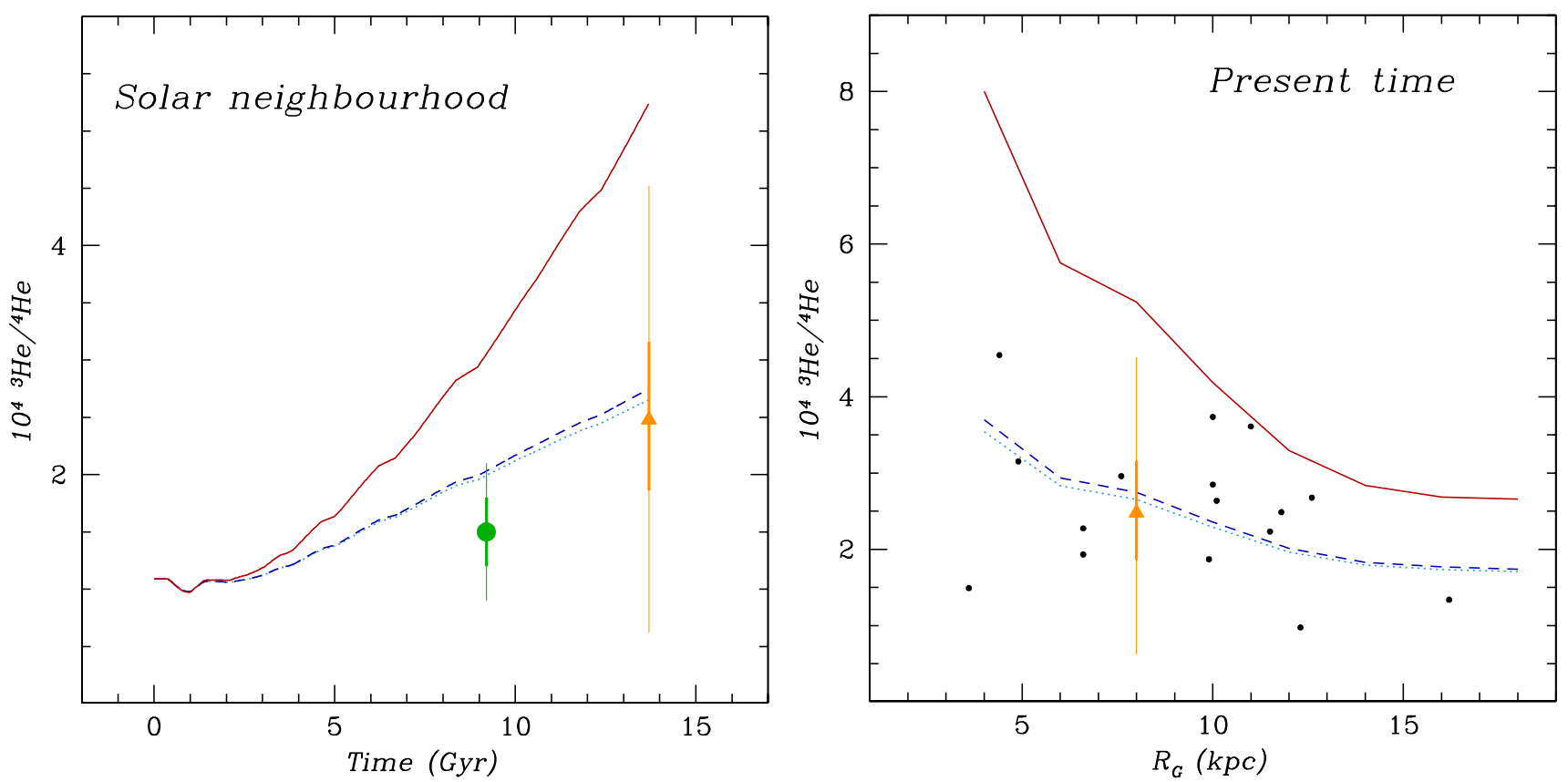

Fig. 8. Temporal (left panel) and spatial (right panel) variation of helium isotopic ratio in the solar neighborhood and along the galactic disk at the present time, respectively. Models are the same as in Fig. 7. Data are from Geiss \& Gloeckler (1998) for the PSC (green filled circle), Bania et al. (2002) for Galactic HII regions (black dots), and Gloeckler \& Geiss (1996) for the LISM (orange filled trangle). 1- $\sigma$ and 3- $\sigma$ errors are also shown as thick and thin lines, respectively.

${ }^{3} \mathrm{He}$ in the stellar interior - and the rotation-induced mixing which increases ${ }^{4} \mathrm{He}$ at the stellar surface (see Sect. 3.3) - are taken into account, lower helium isotopic ratios are obtained (cf. the dashed versus solid lines in Fig. 8). Consequently, the gradient of the helium isotopic ratio across the Galactic disc decreases and the observations in HII regions can be reproduced.

Figure 9 presents the predictions for $Y$ versus $[\mathrm{Fe} / \mathrm{H}]$ in the solar neighborhood. The model predictions are compared with the initial abundance of ${ }^{4} \mathrm{He}$ in the Sun derived by Serenelli (2010) from full standard solar models computed for different sets of solar abundances. When the solar abundances from Grevesse \& Sauval (1998) and a metal to hydrogen ratio in the solar photosphere of $(Z / X)_{\mathrm{ph}}=0.0229$ are adopted, a value of $Y_{i}=0.2721$ is obtained, which results in good agreement with the predictions of chemical evolution models including rotationinduced mixing and thermohaline mixing (green Sun symbol and dashed and dotted lines in Fig. 9, respectively). On the other hand, choosing the solar abundances from Asplund et al. (2009) and $(Z / X)_{\mathrm{ph}}=0.0178$ result in $Y_{i}=0.2653$, which is better fitted by models including standard stellar nucleosynthesis (orange Sun symbol and solid line in Fig. 9). Since the actual solar chemical composition is matter of debate, we can not discriminate between our models basing on the results for $Y$.

\section{Conclusions}

In this article, we have described the results for the Galactic evolution of the primordial elements $\mathrm{D},{ }^{3} \mathrm{He}$ and ${ }^{4} \mathrm{He}$ in the light of new stellar models including the effects of thermohaline instability and rotation-induced mixing on the stellar structure, evolution and nucleosynthesis. The new stellar models are presented and discussed at length in Charbonnel \& Lagarde (2010) and Lagarde et al. (2011). We model the time-behavior of D, ${ }^{3} \mathrm{He}$ and ${ }^{4} \mathrm{He}$ in the solar neighbourhood, as well as in the inner and outer disc. The predictions of our models including thermohaline instability and rotation-induced mixing are compared

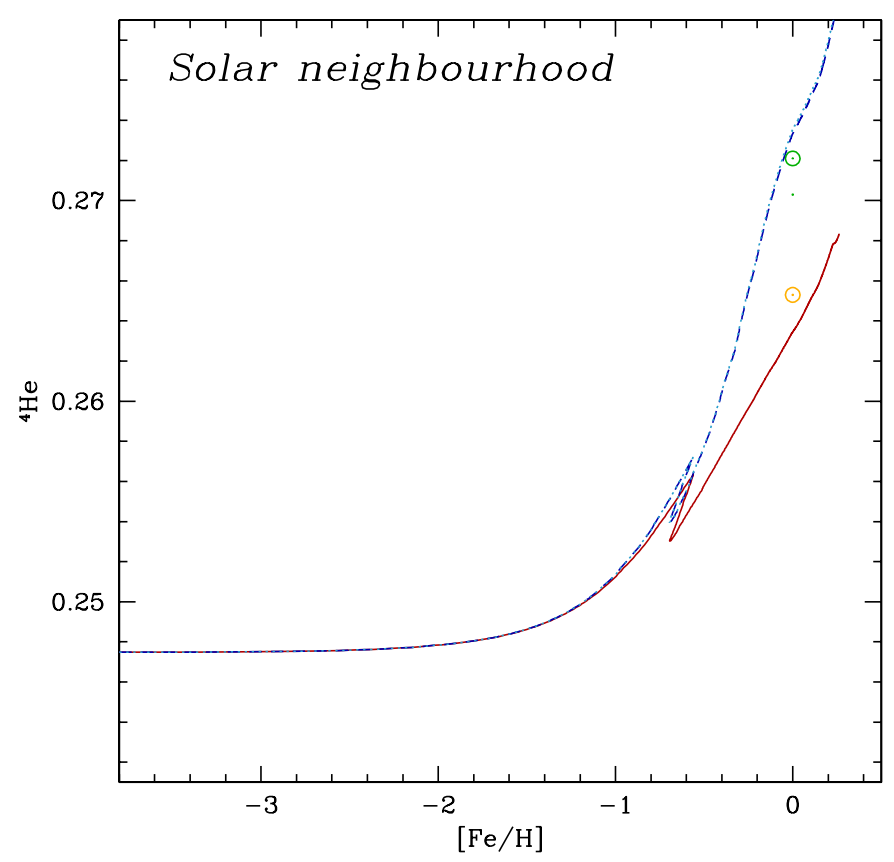

Fig. 9. $Y$ versus $[\mathrm{Fe} / \mathrm{H}]$ in the solar neighborhood predicted by models including yields computed with standard prescriptions (Model A; solid line) and with thermohaline mixing and rotation-induced mixing (Models B and C; dashed and dotted lines, respectively). Model predictions are compared with the protosolar values given by Serenelli (2010).

to the predictions of models adopting standard nucleosynthesis prescriptions and to the most recent relevant observations.

We have seen that the CBP proposed before by Boothroyd \& Sackmann (1999) is not a physical mechanism, and yields deduced do not take into account phases beyond the RGB. As discussed in the literature (Charbonnel \& Zahn 2007b; Charbonnel \& Lagarde 2010; Lagarde et al. 2011), thermohaline mixing can 
induces significant depletion of ${ }^{3} \mathrm{He}$ in low- and intermediatemass stars. Although these stars remain net producers of ${ }^{3} \mathrm{He}$, their contribution to the Galactic evolution of this element is highly reduced compared to classical theory. Indeed, our GCE models including thermohaline mixing reproduce the observations of ${ }^{3} \mathrm{He}$ in the PSC, LISM and HII regions, while ${ }^{3} \mathrm{He}$ is overproduced on a Galactic scale with standard models. Thermohaline mixing is the only physical mechanism known so far able to solve the so-called "3e problem" plaguing in the literature since many years. Importantly, its inhibition by a fossil magnetic field in red giant stars that are descendants of Ap stars does reconcile the measurements of ${ }^{3} \mathrm{He} / \mathrm{H}$ in Galactic HII regions with high values of ${ }^{3} \mathrm{He}$ in a couple of planetary nebulae.

On the other hand, rotation has an impact on the stellar yields of $\mathrm{H}$ and ${ }^{4} \mathrm{He}$. Whether GCE models including rotation fit better the Galactic ${ }^{4} \mathrm{He}$ data than standard models can not be said, because of the uncertainties on the actual solar chemical composition. However, they are consistent with the relatively high value of $(\mathrm{D} / \mathrm{H})_{\text {LiSM }}$ proposed by Prodanović et al. (2010).

We conclude that GCE models including both thermohaline mixing and rotation-induced mixing reproduce satisfactorily well all the available data on D, ${ }^{3} \mathrm{He}$ and, possibly, ${ }^{4} \mathrm{He}$ abundances in the Milky Way, within the errors. However, the additional data on HII regions and better refining of their interpolation would be crucial to assess how good our fit actually is.

Acknowledgements. We dedicate this paper to Robert T. Rood: the scientist to whom the stellar evolution and ${ }^{3} \mathrm{He}$ communities owe so much, and the friend whom we will miss for ever. We thank T. Bania, D. Balser, and H. Reeves for fruitful discussions. We are thankful to our referee, Dr Achim Weiss, for his interesting and constructive remarks on our article. C.C., D.R., and M.T. gratefully acknowledge the enlightening and fascinating conversations with Johannes Geiss and his hospitality at the International Space Science Institute (ISSI) in Bern $(\mathrm{CH})$. We acknowledge financial support from the Swiss National Science Foundation (FNS) and the french Programme National de Physique Stellaire (PNPS) of CNRS/INSU.

\section{References}

Asplund, M., Grevesse, N., Sauval, A. J., \& Scott, P. 2009, ARA\&A, 47, 481 Balser, D. S., Bania, T. M., Rood, R. T., \& Wilson, T. L. 1999, ApJ, 510, 759 Balser, D. S., Goss, W. M., Bania, T. M., \& Rood, R. T. 2006, ApJ, 640, 360 Balser, D. S., Rood, R. T., \& Bania, T. M. 2007, Science, 317, 1171

Balser, D., Rood, T. R., \& Bania, T. M. 2010, in IAU Symp. 268, ed. C. Charbonnel, M. Tosi, F. Primas, \& C. Chiappini, 101

Bania, T. M., Rood, R. T., \& Balser, D. S. 2002, Nature, 415, 54

Bania, T. M., Rood, R. T., \& Balser, D. S. 2010, in IAU Symp. 268, ed.

C. Charbonnel, M. Tosi, F. Primas, \& C. Chiappini, 81

Bennett, C. L., Halpern, M., Hinshaw, G., et al. 2003, ApJS, 148, 1

Boothroyd, A. I., \& Sackmann, I.-J. 1999, ApJ, 510, 232

Burles, S., \& Tytler, D. 1998, ApJ, 507, 732

Charbonnel, C. 1995, ApJ, 453, L41

Charbonnel, C., \& Do Nascimento Jr., J. D. 1998, A\&A, 336, 915

Charbonnel, C., \& Lagarde, N. 2010, A\&A, 522, A10

Charbonnel, C., \& Zahn, J. 2007a, A\&A, 476, L29

Charbonnel, C., \& Zahn, J.-P. 2007b, A\&A, 467, L15

Chiappini, C., Matteucci, F., \& Gratton, R. 1997, ApJ, 477, 765

Chiappini, C., Matteucci, F., \& Romano, D. 2001, ApJ, 554, 1044

Chiappini, C., Renda, A., \& Matteucci, F. 2002, A\&A, 395, 789

Coc, A., Vangioni-Flam, E., Descouvemont, P., Adahchour, A., \& Angulo, C. 2004, ApJ, 600, 544

Costa, E., \& Frogel, J. A. 1996, AJ, 112, 2607

Cyburt, R. H., Fields, B. D., \& Olive, K. A. 2008, J. Cosmology Astropart. Phys., 11,12

Dearborn, D. S. P., Steigman, G., \& Tosi, M. 1996, ApJ, 465, 887

Denissenkov, P. A. 2010, ApJ, 723, 563

Denissenkov, P. A., \& Merryfield, W. J. 2011, ApJ, 727, L8

Edmunds, M. G. 1994, MNRAS, 270, L37

Eggleton, P. P., Dearborn, D. S. P., \& Lattanzio, J. C. 2006, Science, 314, 1580
Eggleton, P. P., Dearborn, D. S. P., \& Lattanzio, J. C. 2008, ApJ, 677, 581

Ekström, S., Meynet, G., Chiappini, C., Hirschi, R., \& Maeder, A. 2008, A\&A, 489, 685

Epstein, R. I., Lattimer, J. M., \& Schramm, D. N. 1976, Nature, 263, 198

Frogel, J. A., Mould, J., \& Blanco, V. M. 1990, ApJ, 352, 96

Galli, D., Palla, F., Ferrini, F., \& Penco, U. 1995, ApJ, 443, 536

Galli, D., Stanghellini, L., Tosi, M., \& Palla, F. 1997, ApJ, 477, 218

Geiss, J., \& Gloeckler, G. 1998, Space Sci. Rev., 84, 239

Geiss, J., \& Reeves, H. 1972, A\&A, 18, 126

Geiss, J., \& Reeves, H. 1981, A\&A, 93, 189

Gloeckler, G., \& Geiss, J. 1996, Nature, 381, 210

Grevesse, N., \& Sauval, A. J. 1998, Space Sci. Rev., 85, 161

Groenewegen, M. A. T. 1998, Ap\&SS, 255, 379

Hébrard, G., Lemoine, M., Vidal-Madjar, A., et al. 2002, ApJS, 140, 103

Hébrard, G., Tripp, T. M., Chayer, P., et al. 2005, ApJ, 635, 1136

Hirschi, R. 2007, A\&A, 461, 571

Hirschi, R., Meynet, G., \& Maeder, A. 2005, A\&A, 433, 1013

Hogan, C. J. 1995, ApJ, 441, L17

Iben, Jr., I. 1967, ApJ, 147, 624

Iwamoto, K., Brachwitz, F., Nomoto, K., et al. 1999, ApJS, 125, 439

Jenkins, E. B., Tripp, T. M., Woźniak, P. R., Sofia, U. J., \& Sonneborn, G. 1999, ApJ, 520, 182

Kobayashi, C., Umeda, H., Nomoto, K., Tominaga, N., \& Ohkubo, T. 2006, ApJ, 653, 1145

Krishnamurti, R. 2003, J. Fluid Mech., 483, 287

Lagarde, N., Charbonnel, C., Decressin, T., \& Hagelberg, J. 2011, A\&A, 536, A28

Lagarde, N., Decressin, T., Charbonnel, C., et al. 2012, A\&A, in press, DOI:10.1051/0004-6361/201118331

Larson, R. B. 1976, MNRAS, 176, 31

Linsky, J. L. 2010, in IAU Symp. 268, ed. C. Charbonnel, M. Tosi, F. Primas, \& C. Chiappini, 53

Linsky, J. L., Draine, B. T., Moos, H. W., et al. 2006, ApJ, 647, 1106

Maeder, A., \& Zahn, J.-P. 1998, A\&A, 334, 1000

Marigo, P., Girardi, L., \& Bressan, A. 1999, A\&A, 344, 123

Matteucci, F., \& Francois, P. 1989, MNRAS, 239, 885

Meynet, G., \& Maeder, A. 2002, A\&A, 390, 561

Oliveira, C. M., \& Hébrard, G. 2006, ApJ, 653, 345

Palla, F., Bachiller, R., Stanghellini, L., Tosi, M., \& Galli, D. 2000, A\&A, 355 , 69

Peebles, P. J. E. 1966, ApJ, 146, 542

Peimbert, M., Peimbert, A., Carigi, L., \& Luridiana, V. 2010, in IAU Symp. 268, ed. C. Charbonnel, M. Tosi, F. Primas, \& C. Chiappini, 91

Pettini, M., Zych, B. J., Murphy, M. T., Lewis, A., \& Steidel, C. C. 2008, MNRAS, 391, 1499

Prantzos, N. 1996, A\&A, 310, 106

Prodanović, T., \& Fields, B. D. 2003, ApJ, 597, 48

Prodanović, T., Steigman, G., \& Fields, B. D. 2010, MNRAS, 406, 1108

Reeves, H., Audouze, J., Fowler, W. A., \& Schramm, D. N. 1973, ApJ, 179, 909

Rogers, A. E. E., Dudevoir, K. A., Carter, J. C., et al. 2005, ApJ, 630, L41

Romano, D. 2010, in IAU Symp. 268, ed. C. Charbonnel, M. Tosi, F. Primas, \& C. Chiappini, 431

Romano, D., Tosi, M., Matteucci, F., \& Chiappini, C. 2003, MNRAS, 346, 295

Romano, D., Tosi, M., Chiappini, C., \& Matteucci, F. 2006, MNRAS, 369, 295

Romano, D., Karakas, A. I., Tosi, M., \& Matteucci, F. 2010, A\&A, 522, A32

Rood, R. T., Steigman, G., \& Tinsley, B. M. 1976, ApJ, 207, L57

Rood, R. T., Bania, T. M., \& Wilson, T. L. 1984, ApJ, 280, 629

Rood, R. T., Bania, T. M., \& Wilson, T. L. 1992, Nature, 355, 618

Rosenblum, E., Garaud, P., Traxler, A., \& Stellmach, S. 2011, ApJ, 731, 66

Serenelli, A. M. 2010, Ap\&SS, 328, 13

Songaila, A., Cowie, L. L., Hogan, C. J., \& Rugers, M. 1994, Nature, 368, 599

Sonneborn, G., Tripp, T. M., Ferlet, R., et al. 2000, ApJ, 545, 277

Spergel, D. N., Verde, L., Peiris, H. V., et al. 2003, ApJS, 148, 175

Steigman, G., \& Tosi, M. 1992, ApJ, 401, 150

Steigman, G., Romano, D., \& Tosi, M. 2007, MNRAS, 378, 576

Tosi, M. 2000, in The Light Elements and their Evolution, ed. L. da Silva, R. de Medeiros, \& M. Spite, IAU Symp., 198, 525

Tosi, M., Steigman, G., Matteucci, F., \& Chiappini, C. 1998, ApJ, 498, 226

Traxler, A., Garaud, P., \& Stellmach, S. 2011, ApJ, 728, L29

Truran, J. W., \& Cameron, A. G. W. 1971, Ap\&SS, 14, 179

Ulrich, R. K. 1972, ApJ, 172, 165

Vidal-Madjar, A., Lemoine, M., Ferlet, R., et al. 1998, A\&A, 338, 694

Wagoner, R. V., Fowler, W. A., \& Hoyle, F. 1967, ApJ, 148, 3

Weiss, A., Wagenhuber, J., \& Denissenkov, P. A. 1996, A\&A, 313, 581

Zahn, J.-P. 1992, A\&A, 265, 115 